\title{
TECNOLOGIA DE NANOSATÉLITE PARA MAPEAMENTO E SENSORIAMENTO REMOTO NA AGRICULTURA, DISSEMINAÇÃO E INDÚSTRIA AGROPECUÁRIA.
}

\author{
T. I. Silva ${ }^{1, *} ;$ R. Barbosa ${ }^{1}$; M. H. E. Morais ${ }^{2}$, R. A. Moura ${ }^{1}$ \\ 1 Faculdade de Tecnologia de São José dos Campos - Professor Jessen Vidal \\ Av. Cesare Mansueto Giulio Lattes, 1350 - Eugênio de Melo, São José dos Campos/SP, \\ CEP.: 12247-014, Brasil. Telefone: (12) 3902-9043 \\ 2 Sistemas Espaciais \& Tecnologia - EMSISTI, Rua: Patativa, 81 - Vl. Tatetuba, São José \\ dos Campos-SP, 12200-140, Brasil. Telefone: (12) 3302-9225 \\ *thiago23inacio21@gmail.com
}

\begin{abstract}
RESUMO: Estudos envolvendo imagens por satélites vem sendo utilizado para coletar dados importantes para a agropecuária. No Brasil o investimento em satélites ainda não possui investidores contumazes, a falta de interesse se dá pela falta de incentivo ao fomento tecnológico governamental e no setor privado. Nesse sentido, o objetivo deste artigo é divulgar a implementação de nanosatélites, que além de ser uma inovação tecnológica, permite um investimento menor do que o satélite padrão. O método deste artigo contará com pesquisa literária e montagem de um protótipo próximo ao real de um nanossatélite de coleta de dados. Os resultados foram a ampla aceitação por parte da sociedade acadêmica e menores custos na fabricação. Conclui-se que está inovação incentivou a indústria agropecuária a ser mais competitiva e controlar melhor sua área de atuação, com visão precisa e panorâmica do campo, via satélite, em tempo real.
\end{abstract}

PALAVRAS-CHAVE: Nanossatélite; Tecnologia e Inovação; Imagem por Satélite; Agronegócio.

ABSTRACT: Studies involving satellite images have been used to collect important data for agriculture. In Brazil the investment on satellites does not have frequent investors, the lack of interest is due to the lack of investment on o technological development by governmental agencies and private companies. In this sense, the goal of this article is to disclose the implementation of nanosatellites, which besides being a technological innovation, allows a smaller investment than the standard satellite. The method of this article will feature literary research and assembly of a prototype close to the real one of a data collection nanosatellite. The results were broad acceptance by the academic society and lower manufacturing costs. It is concluded that this innovation has encouraged the farming industry to be more competitive and better control its area of operation, with accurate and panoramic view of the field, via satellite, in real time.

KEYWORDS: Nanosatellite; Technology and Innovation; Satellite Image; Agrobusiness.

\section{INTRODUÇÃO}

A corrida espacial que ocorreu durante o século XX entre Estados Unidos e União Soviética, proporcionou desenvolvimento em muitas áreas de pesquisas e inovação, como viajar a lua e realizar lançamentos de artefatos ao espaço para comunicação, vigilância, estudos científicos, tecnológicos ou do espaço profundo e de outros planetas. (INPE, 2019) Após este marco importante para evolução 


\section{ISSN $2447-5378$}

espacial, países como Japão, China, Índia e a União Europeia colaboram e incentivam o avanço desta exploração, seguidos de países emergentes como o Brasil, que embora tem colaborado menos, possui um histórico espacial importante no planeta, a Figura 1 demostra as conquistas brasileiras ao longo do tempo, mostrando os primeiros satélites que foram lançados (SANTOS, 2018). Porém em 1991 o brasileiro Junior Torres de Castro se torna o primeiro do país a colocar um satélite em orbita e a única pessoa física no mundo a ter um satélite próprio (CASTRO, 2019).

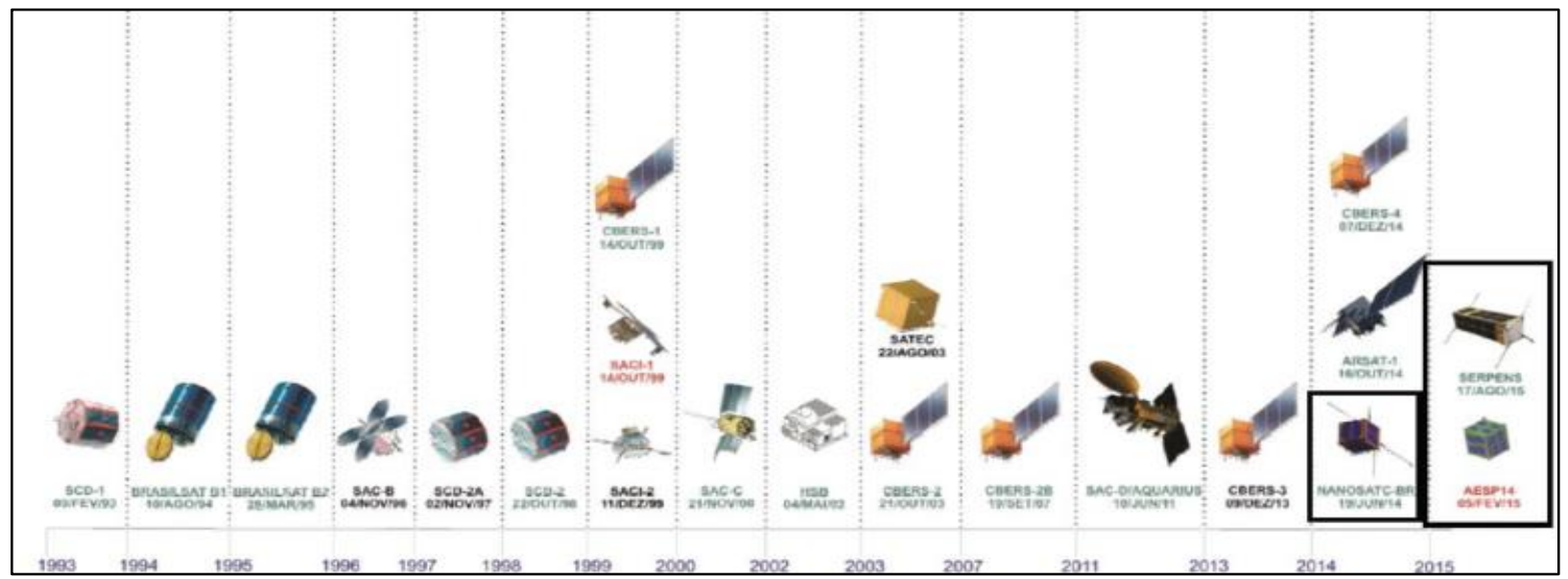

Figura 1. Lançamentos históricos de satélites brasileiros.

Todos os satélites brasileiros que foram lançados obtiveram ajuda externa, ou seja, a capacidade de colocar em órbita por conta própria nunca aconteceu, porem houve tentativas (INPE, 2019). Os problemas político-financeiros brasileiros têm influenciado no avanço das pesquisas espaciais e por este motivo que investimentos em satélites de pequeno porte têm sido comum.

Atualmente os países que mais lançam satélites são disparadamente os Estados Unidos seguido da União Europeia, a Figura 2 mostra os países que estão à frente de investimentos e tecnologia no setor aeroespacial, nota-se que o Brasil está longe de estar entre os primeiros (VILLELA, 2019).

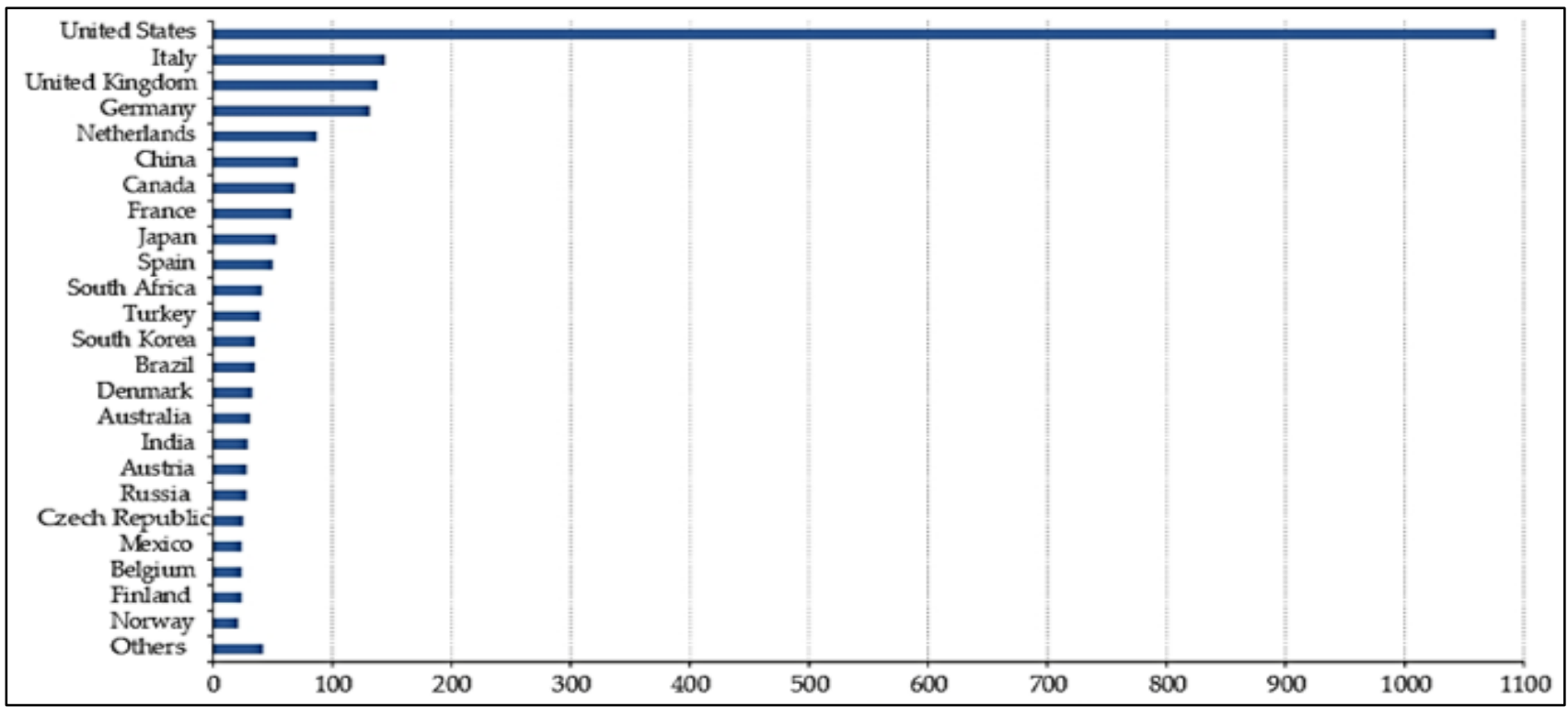

Figura 2. Número de países que investem em tecnologia espacial. 


\subsection{Características de um nanossatélite}

O CubeSat (acrônimo das palavras em Inglês: Cube e Satellite) é um tipo de satélite miniaturizado usado para pesquisas espaciais, tecnológicas, de comunicações radioamadoras e outras que possuem medidas padronizadas que estão indicadas na Figura 3 e massa catalogadas na Tabela 1, pois um CubeSat pode ser considerado um nanossatélite dependendo do tamanho (dimensão) do artefato. Começando em 1999, esforços da California Polytechnic State University liderados por Jordi Puig-Suari e da Universidade Stanford liderados por Bob Twiggs, levaram ao desenvolvimento da especificação do CubeSat com a intenção de ajudar as universidades de todo o mundo a exercer atividades práticas de exploração científica do espaço, incorporando avançados da nanotecnologia, informática, microeletrônica e telecomunicações, entre outras áreas (CAL POLY,2019).

No Brasil o primeiro CubeSat foi desenvolvido pelo INPE, por meio de seu Centro Regional Sul (CRS) em parceria com a Universidade Federal de Santa Maria (UFSM), com apoio da Agência Espacial Brasileiro (AEB), foi lançado em 19 de junho de 2014, a missão do NANOSATC-BR1 apresentada a missão científica de fazer pesquisas sobre clima espacial e fenômenos que impactam a terra, como a Anomalia Magnética do Atlântico Sul (NANOSAT, 2019), a Figura 4 mostra o NANOSATC-BR1 no modelo de engenharia e de voo . Projetado para durar pouco mais de 1 ano, vem surpreendendo parceiros e responsáveis pela coleta de dados, pois após 5 anos encontra-se em atividade e em meados do primeiro semestre de 2020 espera-se lançar o NANOSATC-BR2 (SCHUCH, 2014).

Com o seu tamanho relativamente pequeno, segundo dados da Cal Poly o CubeSat pode ser construído e lançado por um custo estimado de US\$65.000 a US\$80.000. Esse custo, é menor que o de satélites "convencionais" (médio e grande porte), faz do CubeSat uma opção viável para escolas e universidades ao redor do Mundo. Por conta disso, muitas universidades, empresas e até mesmo instituições governamentais, passaram a desenvolver CubeSats (CAL POLY, 2019).

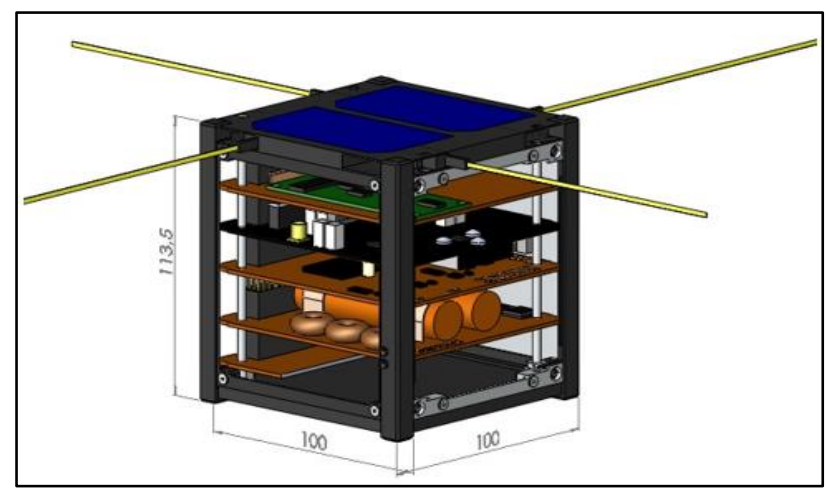

Figura 3. Imagem das medidas de um Nanossatélite. 
Tabela 1. Categoria de satélites por peso.

\begin{tabular}{|l|c|}
\hline \multicolumn{1}{|c|}{ Classe } & Massa (kg) \\
\hline Satélite grande convencional & $>1000$ \\
\hline Satélite pequeno convencional & 500 a 1000 \\
\hline Minissatélite & 100 a 500 \\
\hline Microssatélite & 10 a 100 \\
\hline Nanossatélite & $\mathbf{1}$ a $\mathbf{1 0}$ \\
\hline Picossatélite & $\mathbf{0 , 1}$ a $\mathbf{1}$ \\
\hline
\end{tabular}

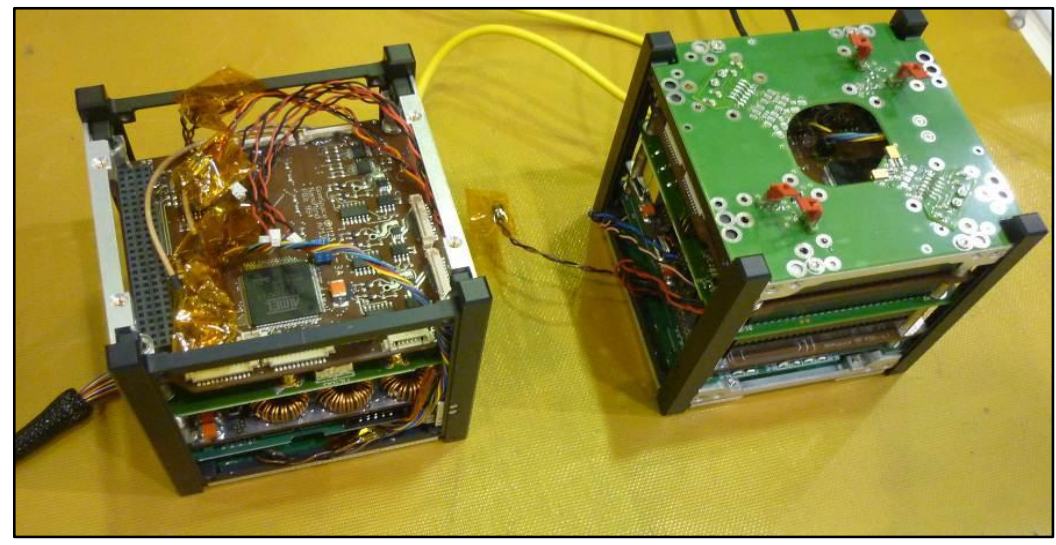

Figura 4. Imagem do primeiro CubeSat brasileiro NANOSATC-BR1. Modelo de Voo e de Engenharia.

O formato padrão de 10×10×10 cm é conhecido como "uma unidade" ou "CubeSat $1 \mathrm{U} "$. Sendo um sistema escalável ao longo de um dos eixos, conforme a Figura 5, nota-se as variações de unidades que se pode ter.

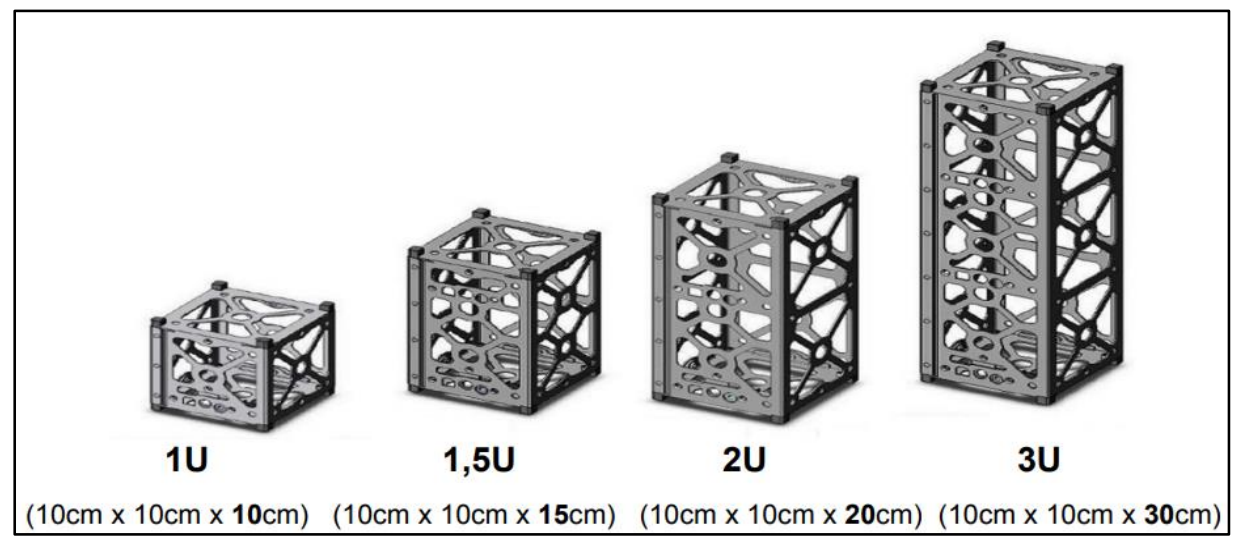

Figura 5. Classificação de unidade de CubeSat.

Embora exista uma padronização de satélites, os mesmos nem sempre respeitam as medidas e o peso, mais recentemente, plataformas maiores vêm sendo propostas, chegando até a "12U" 


\section{ISSN $2447-5378$}

$(24 \times 24 \times 36 \mathrm{~cm})$ estendendo a capacidade além das aplicações acadêmicas e de validação de tecnologias, atingindo objetivos mais complexos nas áreas de ciência e defesa.

Os CubeSats são projetados com componentes comerciais, ou seja, elementos com a confiabilidade baixa e de fácil acessibilidade, isso faz com que o preço para elaboração do projeto fique mais atraente, porém menos confiável, para certificar os componentes o mesmo precisa passar por uma série de teste, que geralmente acontece no LIT (Laboratório de Integração e Testes), localizado na sede do INPE em São José dos Campos-SP.

\section{MATERIAIS E MÉTODOS}

Nesse trabalho foi utilizado um Kit Didático Aeroespacial, modelo CubeSat 1U conforme apresentado na Figura 6, o protótipo seguiu as recomendações básicas de um CubeSat utilizando o barramento I2C. Como base para os simuladores das cargas úteis (CAMARGO, 2018).

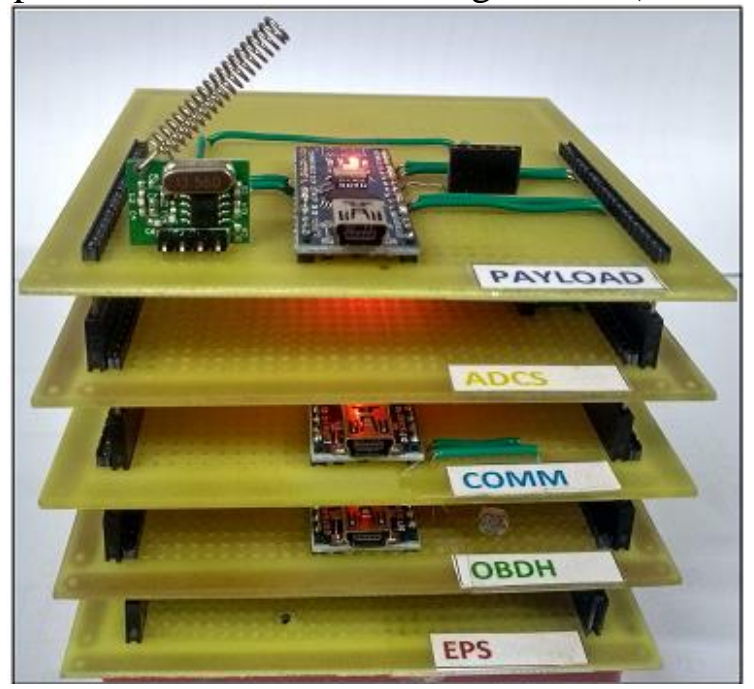

Figura 6. Protótipo de um CubeSat.

O CubeSat é composto por:

a. Uma placa (PAYLOAD): É a placa que integra a carga útil que coleta os dados ambientais.

b. Controle de posicionamento (ADCS): Controla a direção e rotação do nanossatélite.

c. Interface de comunicação (COMM): Que fazem o processamento dos dados internos.

d. Computador de bordo $(\mathrm{OBDH})$ : Responsável por receber dados internos e enviar para estação terrena.

e. Uma placa distribuição de energia (EPS): Mantem a energia e potência de todos os circuitos.

f. Baterias de lítio-íon: Estas baterias são projetadas para alimentar todo o nanossatélite e são capazes de durar vários anos.

g. Módulos solares: O nanossatélite é revestido por placas solares, que são responsáveis por carregar a bateria.

Em uma missão espacial de CubeSat devem respeitar as seguintes fases de projeto com um planejamento distribuído pelas seguintes etapas:

a. Análises da missão: Indica qual a missão que o CubeSat irá realizar. 
b. Análise de viabilidade: Qual tipo de estação solo o CubeSat usará durante sua operação, com qual o tipo de informação irá trabalhar e de qual forma os usuários irão receber os dados.

c. Definição preliminar do projeto: Um CubeSat precisa de no mínimo cinco sistemas, uma carga útil responsável por colher dados (câmera integrada), uma fonte de energia, um computador de bordo, controle de altitude e temperatura interna e estrutura.

d. Definição detalhada do projeto: Definir quais baterias serão utilizadas, a forma em que o computador de bordo vai processar as informações, como o controle e atitude vai monitorar a luz solar, detalhar o controle térmico do sistema interno, indicar como a telemetria vai trabalhar caso o controle térmico falhe e definir a estrutura do CubeSat.

e. Produção e qualificação: Passar por inúmeros teste térmicos e de vibração, geralmente estes testes são realizados no LIT (Laboratório de Integração e Testes).

f. Operação: Lançamento e análise de toda dinâmica envolvida no CubeSat.

g. Descarte: Após o tempo de operação no espaço o CubeSat necessita de um descarte correto para que não seja um lixo espacial.

\subsection{Métodos para Implementação de CubeSat na indústria de Agronegócios}

Com o desenvolvimento de novas tecnologias empresas têm utilizado novos métodos de produção baseados em automação, robótica, internet das coisas dentre outras inovações. No contexto geral, as empresas estão cada vez mais competitivas de modo a otimizar a eficiência da cadeia produtiva. Com a indústria agropecuária não é diferente, pois inovações acontecem a todo momento no campo, estudos envolvendo imagens por satélites vem sendo utilizados para coletar dados importantes para os agricultores. Estas imagens são terceirizadas de satélites internacionais, custando um valor persuasivo ao consumidor, porém no estado atual é mais vantajoso obter imagens de um mediador do que colocar em órbita um satélite (EMBRAPA, 2019).

Os satélites são capazes de obter imagens perfeitas de um terreno ou uma área expressiva. A Figura 7 mostra o campo em alta resolução seguido da Figura 8 que é de média resolução e da Figura 9 com baixa resolução. Missões de Coleta de Dados Ambientais no Agronegócio, surgiu da necessidade de inúmeras empresas e instituições obter regularmente informações colhidas em lugares remotos ou espalhadas por uma região muito extensa.

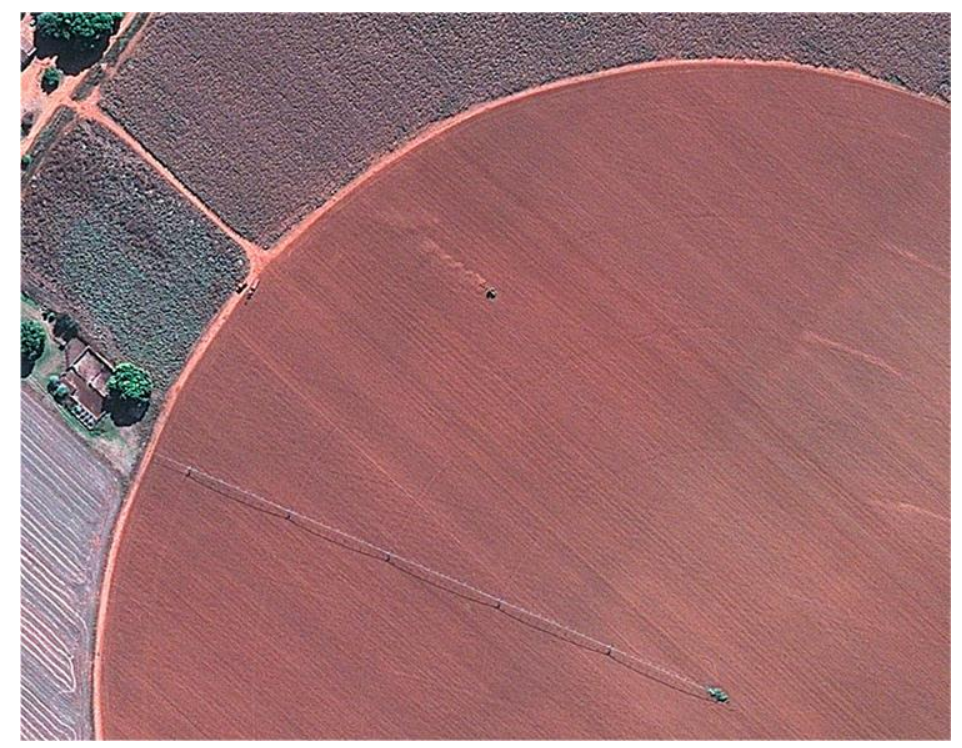

Figura 7.Imagem de alta resolução. 


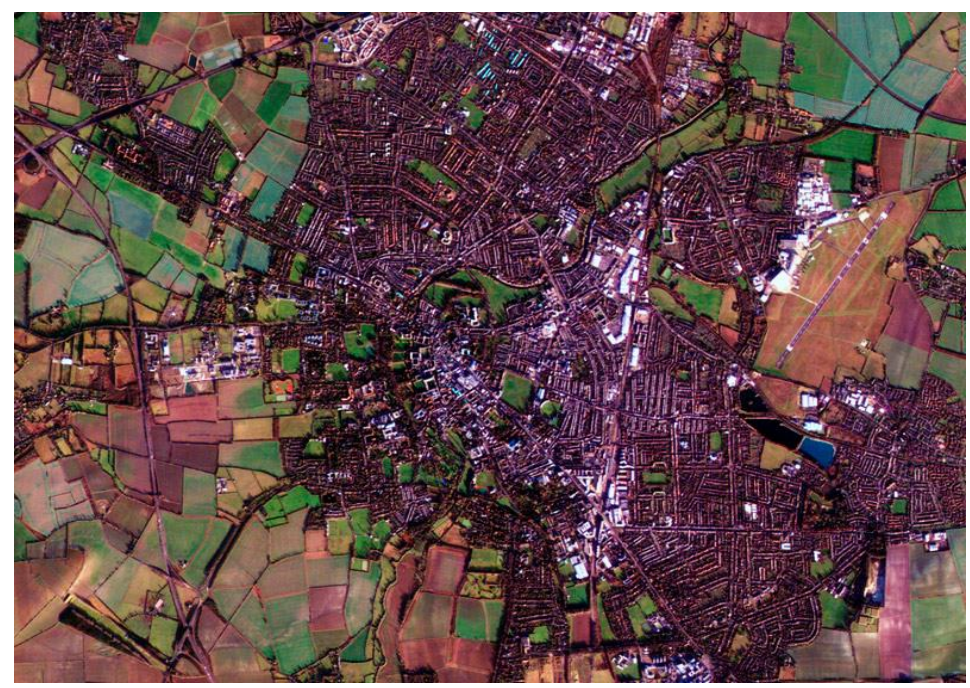

Figura 8. Imagem de média resolução.

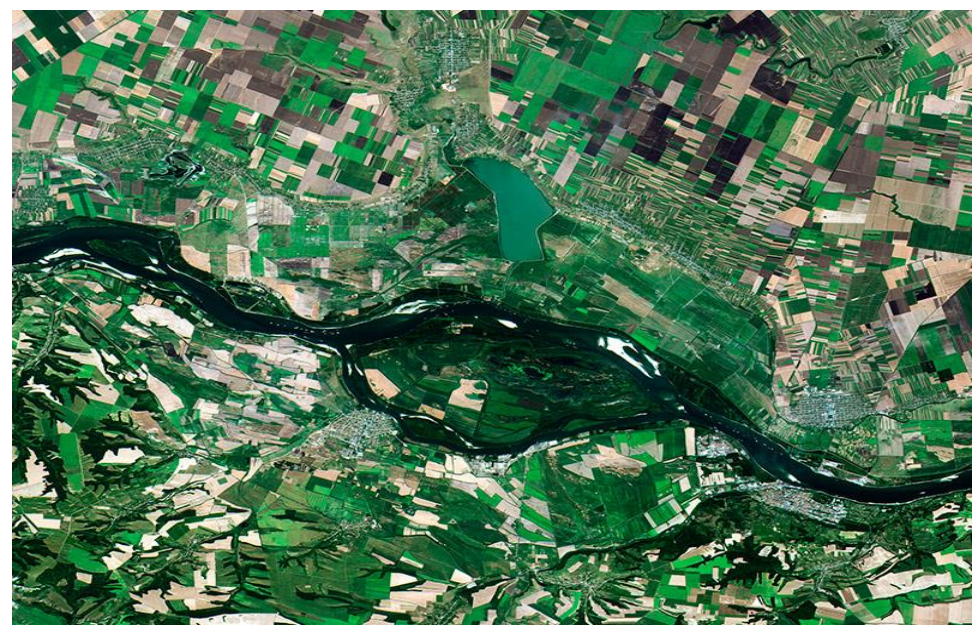

Figura 9. Imagem de baixa resolução.

As figuras foram obtidas da web site da empresa GEOCENTO, que comercializa imagens de satélite. Na Figura 7 a imagens pode custar cerca de US\$200 por $8 \mathrm{~km}^{2}$ com resolução de até $30 \mathrm{~cm}$ do solo, na Figura 8 pode custar aproximadamente US $\$ 100$ por $4 \mathrm{~km}^{2}$ com resolução de $1 \mathrm{~m}$ até $5 \mathrm{~m}$ do solo, na Figura 9 o site não menciona valores, isso porque o valor é menor e a imagem não é atrativa para o cliente que busca uma boa imagem (GEOCENTO, 2019).

Os CubeSats foram criados para atender às necessidades de estudantes, porém com o avanço desta tecnologia podem ser usados em aplicações comerciais. Atualmente aplicados ao agronegócio possui informações valiosas, como por exemplo: controle e monitoramento de criações e plantações, vegetação com muita irrigação ou pouca irrigação e contaminação do solo. Mesmo que o nanossatélite não faça imagens de alta resolução ele pode fazer análise de um campo específico, localizar a irregularidade indicando o local a ser estudado e revela se a necessita de uma imagem de alta ou média resolução, isto faz com que a terceirização da imagem fique mais acessível. A Figura 10 indica a forma que o CubeSat pode atuar na coleta de dados. 


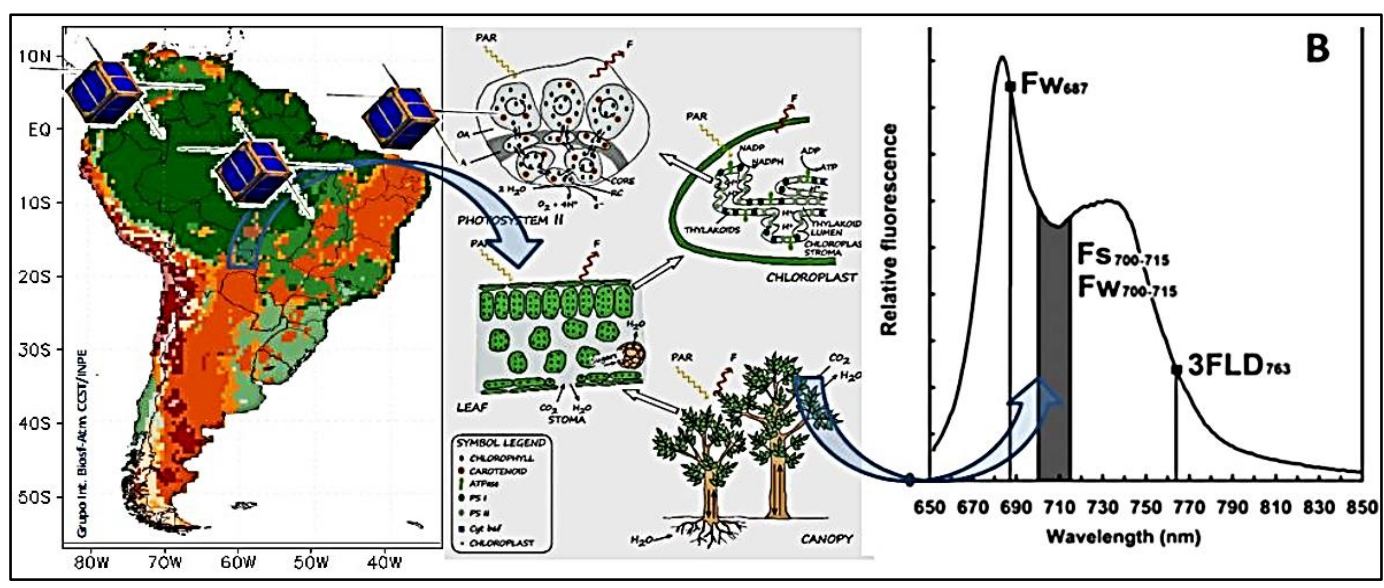

Figura 10. Identificação de pragas e irregularidades na vegetação.

Os satélites, podem retransmitir dados coletados em solo e reenviar para uma ou mais estação terrena, estes dados podem ser analisados conforme a radiação na faixa do infravermelho termal que as plantas emitem quando estão adoecida em seus níveis de maturidade, e isso, por sua vez, processa e gera informações para que o agricultor ou operador possa tomar decisões importantes, como: aonde e quando plantar, em que datas acontecem ou aconteceram irregularidades no terreno e diminuir a quantidade de agrotóxico, pois, com ajuda de fatos (observados, medidos) promove uma melhora na qualidade da colheita dos alimentos. Inclusive, é possível com apenas um computador centralizado comandar diversas máquinas na operação do campo, melhorando o plantio e evitando desperdícios de tempo, fazendo com que a produção seja específica (no local) e atenuada, como indicado na Figura11.

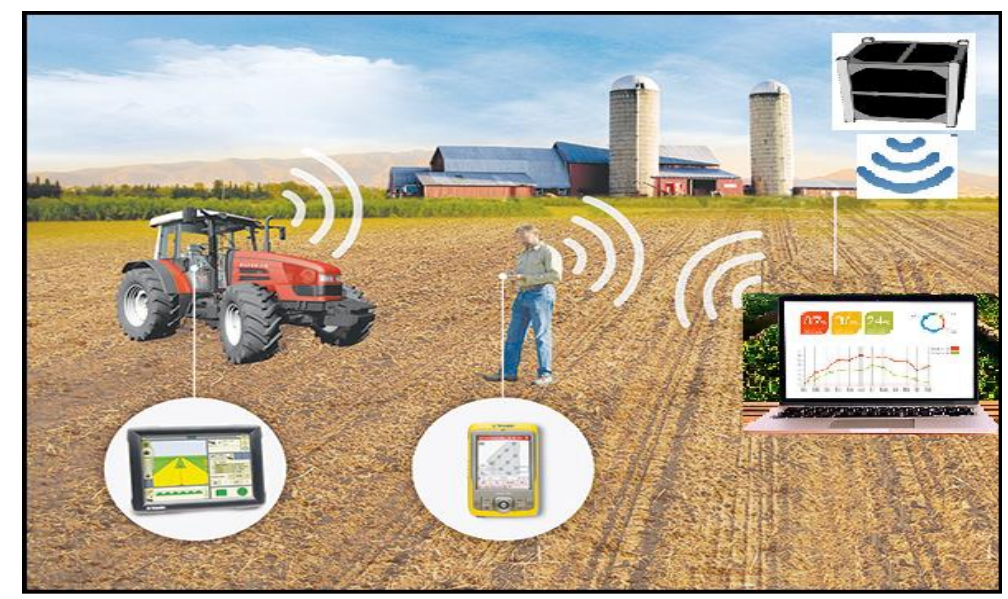

Figura 11. Indica a comunicação entre o CubeSat, estação terrena, o homem e as máquinas.

Com o avanço desta tecnologia, a comunicação entre homem e máquina fica evidente, pois o agricultor pode controlar e monitorar toda a operação em tempo real, melhorando o desenvolvimento dos processos no campo.

\section{RESULTADOS E DISCUSSÕES}

Com o uso do Kit Didático Aeroespacial versão CubeSat, é possível realizar testes de coleta de dados ambientais como: temperatura, umidade do ar, posição geográfica e dados de seus subsistemas. Estes dados podem ser comparados com Estações Ambientais reais utilizadas pelo INPE e INMET 
(Instituto Nacional de Meteorologia). A Figura 12 mostra dados coletados pelo protótipo e que são comparados com dados de uma estação fixa ilustrado na Figura 13, pois a estação é ligada a vários sensores dos parâmetros meteorológicos que integra os valores observados minuto a minuto e os disponibiliza automaticamente a cada hora (INMET, 2019).

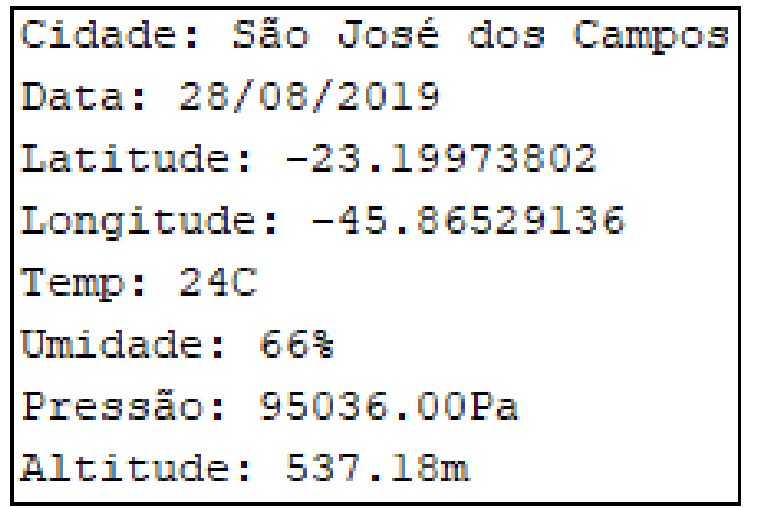

Figura 12. Dados coletados pelo protótipo no município de São José dos Campos.

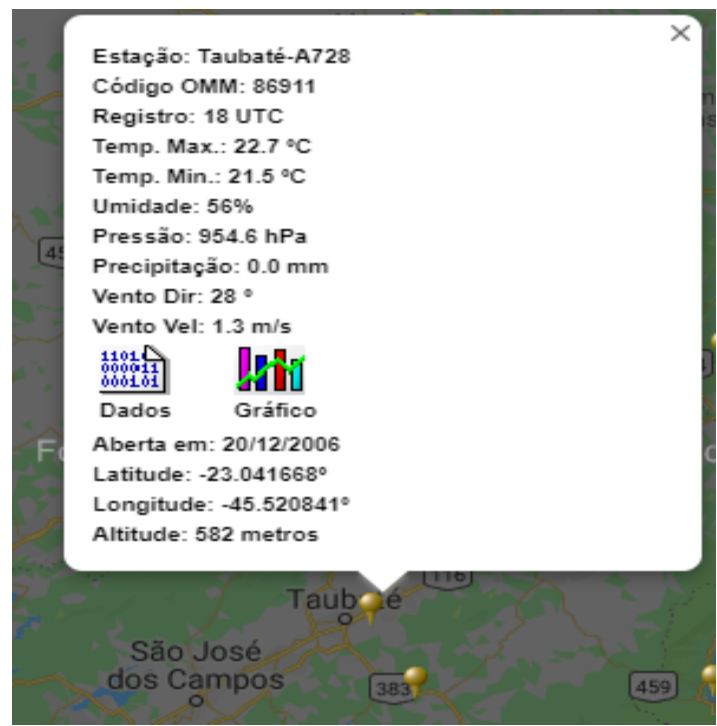

Figura 13. Estação de Coleta de Dados Ambientais, município de Taubaté-SP.

O uso do nanossatélite pode facilitar o trabalho de todos no campo, agricultores, operadores, fazendo com que haja uma comunicação entre satélites, homem e máquinas, isto pode ocorrer de forma direta, sendo possível utilizar apenas algumas pessoas para monitorar os dados e/ou decisões do computador. O objetivo não é retirar o homem do trabalho, mais sim introduzir pessoas mais capacitadas a fazer operações mais complexas, desta forma o processo de trabalho seria automatizado tornando mais rápido e econômico para o produtor e prover qualidade de vida aos colaboradores. Com o avanço dessa tecnologia e com incentivo no agronegócio, indústrias poderão ser mais competitivas, melhorando e desenvolvendo novos processos entre o campo e o espaço.

\section{CONCLUSÃO}

Este é um artigo tecnológico que busca oferecer contribuição para a disseminação da aplicação de técnicas na solução de problemas nas áreas de tecnologia e do agronegócio, apresentando 


\section{ISSN $2447-5378$}

aplicações de melhoria incremental no eixo tecnológico no campo. Com base nas pesquisas realizadas a importância de nanossatélites no mapeamento possibilitou uma condição de trabalho mais efetiva onde é possível diagnosticar possíveis variações no solo e no clima terrestre das regiões para verificar quais os tipos de cultivos ou criação de animais será mais adequada para cada território analisado e fazendo com está tecnologia se torne uma excelente ferramenta na produção de alimentos, cultivos de plantas e monitoramento do campo em geral. Com a captura de imagens do território a ser estudado possibilita uma varredura e com os dados estatísticos será possível prever variações no sistema climático da região.

Com base no experimento realizado de coleta de dados ambientais, foi realizado uma comparação entre as informações retiradas do site do INMET e do protótipo, nota-se a variação de temperatura, umidade e pressão entre uma cidade e outra, com estes dados sendo gerados simultaneamente podem ajudar na produtividade da plantação, fazendo com que o monitoramento dessas variáveis seja de suma importância para uma melhor tomada de decisão no campo, pois o registro a longo prazo dessas variáveis fornece suporte para um planejamento agrícola mais eficaz.

Já, tomando como base os dados nos artigos pesquisados observou-se a necessidade de aumentar o número de profissionais na área, pois o Brasil ainda não possui estrutura que atenda a demanda destes cuidados, existindo uma verdadeira lacuna no processo de captura de dados. $\mathrm{O}$ sensoriamento remoto do território faz com que o monitoramento nas áreas de atuação seja precisa e panorâmica do campo, via satélite. E que com o desenvolvimento do país essa tecnologia precisa ser implantada no meio empresarial, pois a solução para o desenvolvimento nesta área de atuação só será possível com investimentos em sensoriamento remoto.

Trabalhos futuros indicam o uso desta tecnologia para auxiliar ações de segurança nas fronteiras terrestres, aéreas marítimas e na prevenção de propagação de doenças e pragas.

\section{AGRADECIMENTOS}

Os autores agradecem o apoio da FAPESP (Fundação de Amparo à Pesquisa do Estado de São Paulo), processo número 2016/5158-8, da FINEP (Financiadora de Estudos e Projetos), processo número 2016/50514-9 e da EMSISTI Sistemas Espaciais \& Tecnologia pelo suporte financeiro, materiais e métodos.

\section{REFERÊNCIAS}

CAL POLY. Valores de lançamento e características de um CubeSat. Disponível em http://www.polysat.org/. Acesso em: 28 de maio de 2019.

CAMARGO, L., ABRAHÃO, W. Uma proposta de EGSE para o CubeSat NanosatC-BR2. 9. Workshop em Engenharia e Tecnologia Espaciais. INPE, São José dos Campos, 15 e 16 de agosto de 2018.

CASTRO, J. T. Idealizador do Satélite DOVE na frequência de rádio PY2BJO. Única pessoa física no mundo a ter um satélite próprio. Disponível em https://www.qsl.net/py4zbz/py2bjo.htm. Acesso em 27 de agosto 2019 .

EMBRAPA. Satélite brasileiro de alta resolução vai aprimorar monitoramento agrícola. Disponível em https://www.embrapa.br/busca-de-noticias/-/noticia/40022384/satelite-brasileiro-de-altaresolucao-vai-aprimorar-monitoramento-agricola. Acesso em: 25 de maio de 2019. 
GEOCENTO. Valor de imagens retiradas de um Satélite. Disponível em http:// http://geocento.com/imagery-pricing-plans/. Acesso em: 15 de maio de 2019.

INPE. $\quad$ A origem do INPE na corrida espacial. Disponível em http://www.inpe.br/institucional/sobre_inpe/historia.php. Acesso em: 05 de maio de 2019.

INMET. Estação Meteorológica de Observação de Superfície Automática. Disponível em http://www.inmet.gov.br/portal/index.php?r=estacoes/estacoesAutomaticas. Acesso em: 28 de agosto de 2019.

NANOSAT. Primeira missão espacial brasileira com o uso de CubeSats. Disponível em http://www.inpe.br/crs/nanosat/. Acesso em: 12 de maio de 2019.

SANTOS, W. A. O Desenvolvimento de CubeSats no Brasil. SeCIAer2018 - Seminário Serviços Científicos - INPE - 02 de outubro de 2018.

SCHUCH, N. J.; DURÃO, O. S. C. The Capacity Building of the INPE-UFSM NANOSATC-BR, CubeSat Development Program The NANOSATC-BR1 Launching at Yasny - Russia. V Simpósio Brasileiro de Geofísica Espacial e Aeronomia Natal/RN - 29 de setembro a 03 de outubro de 2014.

VILLELA T.; COSTA C. A.; BRANDÃO A. M.; BUENO F. T.; LEONARDI R. Towards the Thousandth CubeSat: A Statistical Overview - International Journal of Aerospace Engineering Volume 2019 - 10 janeiro 2019. 